\title{
Identification of a new missense mutation in MyBP-C associated with hypertrophic cardiomyopathy
}

\author{
Johanna C Moolman-Smook, Bongani Mayosi, Paul Brink, Valerie A Corfield
}

\begin{abstract}
Hypertrophic cardiomyopathy is a primary cardiac disease, characterised by idiopathic myocardial hypertrophy, and is caused by defects in sarcomeric protein encoding genes. One of these genes is cardiac myosin binding protein C (MyBP-C), in which a number of splice site and duplication mutations causing HCM have been described. During mutation screening of a South African HCM population by PCR-SSCP, a missense mutation, Arg654His, was detected in one proband. Although the mutation was present in his three adult children, only the proband himself was markedly affected. This is the first report of a disease associated missense mutation in MyBP-C which does not affect the myosin or titin binding domains.

( $¥$ Med Genet 1998;35:253-254)
\end{abstract}

Keywords: myosin binding protein C; missense mutation; hypertrophic cardiomyopathy

University of

Stellenbosch and

South African Medical

Research Council

Centre for Molecular

and Cellular Biology,

Department of

Medical Physiology

and Biochemistry,

University of

Stellenbosch,

Tygerberg, South

Africa

J C Moolman-Smook

V A Corfield

Cardiac Clinic, Department of Medicine, University

of Cape Town and

Groote Schuur

Hospital, Cape Town,

South Africa

B Mayosi

Department of

Internal Medicine,

University of

Stellenbosch,

Tygerberg, South

Africa

P Brink

Correspondence to:

Dr Corfield.

Received 29 May 1997 Revised version accepted for publication 5 September 1997
Hypertrophic cardiomyopathy (HCM) is a disease of the sarcomere manifesting as a primary cardiac disorder, characterised by myocardial hypertrophy, especially of the interventricular septum, and myocytic and myofibrillar disarray. ${ }^{1}$ It also carries an increased risk of sudden death, a phenomenon which mostly affects the under 40 years age group. ${ }^{2}$ Both the familial (FHC) and sporadic forms of this disease have been associated with mutations in various sarcomeric protein encoding genes, such as the cardiac $\beta$-myosin heavy chain gene, ${ }^{3}$ troponin $T,{ }^{1} \alpha$-tropomyosin, ${ }^{1}$ the essential and regulatory myosin light chains, ${ }^{4}$ and myosin binding protein $\mathrm{C}$ (MyBP-C). ${ }^{56}$

During mutation screening of $\mathrm{MyBP}-\mathrm{C}$ in a panel of HCM affected South Africans, using polymerase chain reaction (PCR) based single stranded conformation polymorphism (SSCP) analysis, a missense mutation was detected in one person of mixed racial ancestry. Previously reported FHC causing mutations in MyBP-C have involved two splice sites ${ }^{56}$ and an exonic duplication event. ${ }^{6}$

Human genomic DNA isolated from peripheral lymphocytes was PCR amplified using the published primers F-intron and R-intron, which amplify domain C5 of MyBP-C, and reported temperature profiles ${ }^{5}$ in a standard reaction mixture. ${ }^{7}$ Subsequently, PCR-SSCP analysis was performed on $5 \%$ polyacrylamide gels as previously described. ${ }^{7}$ Direct single stranded sequencing using a standard protocol ${ }^{8}$ showed that the mobility shift observed in HCM affected subject SB812 was the result of a $G$ to $A$ transition at the second base of codon 654 of the gene (fig 1). ${ }^{9}$ This change leads to the substitution of the wild type arginine at this position in the protein by histidine. Fortuitously, the change also results in the loss of an Fnu4HI site. Cleavage of the approximately 367 bp amplified fragment with Fnu4HI generates three fragments of $\sim 170, \sim 110$, and $\sim 87 \mathrm{bp}$ in size in the absence of the mutation, and an additional $\sim 257 \mathrm{bp}$ fragment in its presence. This direct diagnostic test was applied to a panel of 100 unrelated controls of mixed ancestry, as well as to the family of SB812 (pedigree 136).

In SB812, the $\sim 170, \sim 110$, and $\sim 87$ bp fragments, as well as the $\sim 257$ bp fragment, were present after Fnu4HI digestion, indicating heterozygosity at codon 654 . The mutation was also present in three of the four children of SB812; however, its penetrance was low. In clinical studies, performed as previously described, ${ }^{8}$ the 60 year old proband presented with chest pain and palpitations, he showed a maximum end diastolic septal thickness of 21.2 $\mathrm{mm}$ on echocardiography, abnormal $\mathrm{Q}$ waves and repolarisation features on electrocardiogram (ECG), and periods of sustained monomorphic ventricular tachycardia were detected. In addition, he had sustained an earlier cerebrovascular accident. However, on echocardiography only one of his mutation carrier offspring displayed evidence of mild hypertrophy, a 30 year old daughter with a maximum septal thickness of $11 \mathrm{~mm}$. Septal thicknesses in the other two carriers, who were 33 and 30 years old, respectively, were well within the normal range, while the 33 year old sib showed increased voltages on ECG, but no $P$ wave or repolarisation changes consistent with ventricular hypertrophy. The earlier generation relatives of SB812 and his sibs were dead. The mutation was not detected in unrelated controls.

The MyBP-C protein consists of repetitive modules with homology to either immunoglobulin (Ig)-like or fibronectin type 3 domains. ${ }^{9}$ The previously reported splice site and duplication FHC causing mutations are presumed to alter the carboxyterminal myosin or titin binding modules of MyBP-C. ${ }^{10}{ }^{11}$ The present study is the first report of a disease associated missense mutation occurring in domain C5, a region of MyBP-C not directly associated with these primary binding 


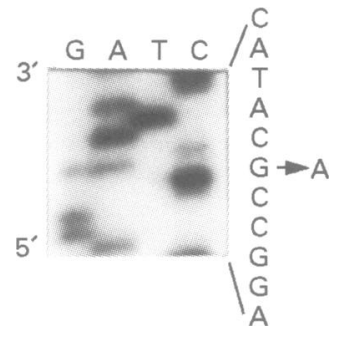

Figure 1 Partial sequence of $M y B P-C$ in $S B 812$, showing the heterozygous $G$ to $A$ transition which is responsible for Arg654His. domains, and indicates the functional importance of another region of the molecule.

Structurally similar amino acid residues are found at position 654 in different MyBP-C isoforms, for example, the polar residues arginine and lysine in human cardiac MyBP-C and human and chick fast MyBP-C, respectively. ${ }^{9}$ As a result of the mutation described, a residue with a basic long side chain is replaced by histidine, in which the side chain consists of an amidazole ring structure with only weakly basic properties. Amino acid 654 occurs in a proline charged residue rich region of MyBP-C, characteristic of motifs which often form the basis of specific ligand interactions of signal transduction molecules. ${ }^{9}$ The residue is also situated near the beginning of the Ig-like domain of module $\mathrm{C} 5$, which is bisected by a unique, cardiac specific insertion sequence, previously postulated to act as a scaffold for the binding of the MyBP-C associated $\mathrm{Ca} 2+/$ calmodulin kinase II. $^{9}$ Consequently, it may be proposed that the Arg654His substitution will have detrimental effects on signal transduction, hindering MyBP-C's phosphorylation dependent adrenergic regulation of cardiac contraction.

Alternatively, as the $100 \mathrm{kDa}$ proteolytic fragment which stretches from the MyBP-C motif to module C9 of MyBP-C, including module $\mathrm{C} 5$, does play some role in myosin MyBP-C binding, ${ }^{10}$ the Arg654His MyBP-C mutation may destabilise such interaction as it conceivably also disrupts the Ig-like domain.

This novel Arg654His MyBP-C mutation is presumed to be responsible for FHC. It was found in an affected subject, in whom extensive SSCP and restriction enzyme allele specific mutation analysis of known HCM causing genes showed no other sequence variation. Furthermore, MyBP-C Arg654His, which was not detected in a panel of controls, codes for an amino acid residue, in which side chain length and charge is conserved. In the small family studied, the mutation showed low penetrance and further studies are needed to determine the role of the Arg654His mutation in MyBP-C related FHC.

This study was supported by the Medical Research Council of South Africa and the University of Stellenbosch.

1 Thierfelder L, Watkins H, MacRae C, et al. $\alpha$-Tropomyosin and cardiac troponin $\mathrm{T}$ mutations cause familial hypertrophic cardiomyopathy: a disease of the sarcomere. Cell 1994;77:701-12.

2 Liberthson RR. Sudden death from cardiac causes in children and young adults. $N$ Engl $\mathcal{F}$ Med 1996;334:1039-

3 Vikstrom KL, Leinwand LA. Contractile protein mutations and heart disease. Curr Opin Cell Biol 1996;8:97-105.

4 Poetter K, Jiang H, Hassanzaadeh S, et al. Mutations in either the essential or regulatory light chains of myosin are associated with a rare myopathy in human heart and skeletal muscle. Nat Genet 1996;13:63-9.

5 Bonne G, Carrier L, Bercovici J, et al. Cardiac myosin binding protein-C gene splice acceptor site mutation is associated with familial hypertrophic cardiomyopathy. $\mathrm{Nat}$ Genet 1995;11:438-40.

6 Watkins $\mathrm{H}$, Conner D, Thierfelder J, et al. Mutations in the cardiac myosin binding protein-C gene on chromosome 11 cause familial hypertrophic cardiomyopathy. Nat Genet cause familial hyp

7 Moolman JC, Brink PA, Corfield VA. Identification of a new missense mutation at Arg403, a CpG hotspot, in exon 13 of the $\beta$-myosin heavy chain gene in hypertrophic cardiomyopathy. Hum Mol Genet 1993;2:1731-2.

8 Posen BM, Moolman JC, Corfield VA, Brink, PA. Clinica and prognostic evaluation of familial hypertrophic cardiomyopathy in two South African families with differen $\beta$-myosin heavy chain gene mutations. Br Heart $¥ 1995 ; 74$ : $40-6$.

9 Gautel M, Zuffardi O, Freiburg A, Labeit S. Phosphorylation switches specific for the cardiac isoform of myosin binding protein-C: a modulator of cardiac contraction? EMBO F 1995;14:1952-60.

10 Okagi T, Weber FE, Fischman DA, Vaughan KT, Mikawa T, Reinach FC. The major myosin-binding domain of skeletal muscle MyBP-C (C protein) resides in the COOHmuscle MyBP-C (C protein) resides in the COOH123:619-26.

11 Freiberg A, Gautel M. A molecular map of the interactions between titin and myosin-binding protein-C. Implications for sarcomeric assembly in familial hypertrophic cardiomyopathy. Eur ₹ Biochem 1996;235:317-23. 